\title{
Fractal Complexity in Built and Game Environments
}

\author{
Daniel Della-Bosca, Dale Patterson, and Sean Costain \\ Griffith University, Gold Coast, Australia \\ \{d.della-bosca, d.patterson, s.costain\}@griffith.edu.au
}

\begin{abstract}
Fractal patterns provide an automated mathematical method to create rich and engaging visuals. These methods have been applied in the design of physical and game spaces to only a limited extent. The current physical and virtual game worlds are dominated by rectangles, squares and linear concepts. This research studied the nature of fractal patterns and in particular the use of differing levels of fractal complexity to design physical and virtual environments. The findings from the randomized trial identified differing levels of fractal complexity and their aesthetic appeal to participants. These levels of fractal complexity were then applied to spatial environments in games to create spaces that were more or less appealing to the participant. The principle of using fractal complexity as a design tool to make an environment more or less comfortable provided game and architectural designers an additional mechanism to enhance spaces and levels of participant engagement.
\end{abstract}

Keywords: fractal, surface, complexity, built environment, computer game.

\section{Introduction}

The world of computer games and their interactive environments is one filled with a mixture of differing spatial locations. Much like the real world, each virtual location features structural elements, both man made and natural. Every location and surface is created geometrically and carefully designed to provide players with a particular visual, ideally immersive, experience. The repetitive process, at heart of fractal mathematics, generates engaging, often uncannily natural patterns, images and objects $[1,2,3]$. The potential to use this fractal technique to enhance the surface textures and bump maps in game spaces was the key focus of this project.

In aesthetic terms, 'An object is said to be self-similar if it looks "roughly" the same at any scale' $[1,2]$, an image is called fractal if it displays self-similarity, that is, it can be broken into parts, each of which is (approximately) a reduced size copy of the whole. The father of fractals, Benoit Mandelbrot, summarized his career as the 'ardent pursuit of the concept of roughness.' [1]. It is this "roughness" that creates the link to natural surfaces, spaces and objects, where fractal patterns are comparatively common [1], [3], [4], [5]. Analysis of architectural design also identifies fractal "cascade of details" in many iconic structures such as the Sydney Opera House, Taj Mahal and others, all applying fractal systems to positive impact $[6,7,8]$.

Games design procedural techniques, including fractal systems have been used for content generation to good effect [3],[4],[9]. Aesthetics are less clearly understood. 


\section{Fractal Dimension, Complexity and Design}

The concept of a fractal pattern being self-similar does not inherently require it to be either complex or simple. There exists a range of possible levels of fractal complexity within this mathematical space. For example the 2D fractal dimension (D value) ranges anywhere between 1.0 and 2.0. A pattern that fills the plane in very simplistic terms (a low order of roughness/irregularity) has a $\mathrm{D}$ value close to 1 , and a pattern that fills the plane with detailed and intricate structure will have a D value close to 2 . From a designers perspective this allows the ability to consider the fractal dimension as a changeable variable to alter the visual and aesthetic nature of the fractal content being created. For 3D objects the dimension ranges from 2.0-3.0.

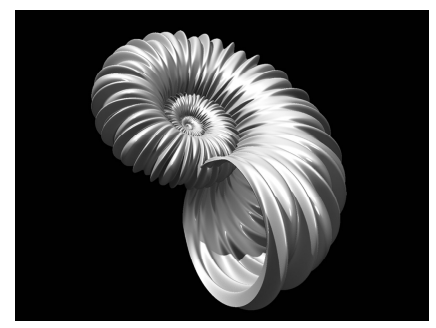

Fig. 1. Early experimental fractal forms from this project demonstrating natural features

Several research studies have measured the aesthetic preferences of participants with regard to images with varying levels of fractal complexity, indicating an average aesthetic preference for a fractal complexity level in the range of 1.3 to $1.5[10,11]$.

\section{Experimental Study of Fractal Complexity}

Earlier studies of fractal dimension and complexity have dealt with mixed imagery from both nature and digital systems, including variations in shape, color and pattern. This research sought to reduce the amount of variables in the imagery studied and to more specifically explore the link between fractal dimension and its aesthetic appeal. The study included twenty-five participants from a range of cultures ranging in age from 18 to 52 years. Each participant was presented with a range of surface options, these surfaces included a range of digitally created fractal surfaces and objects, each with differing levels of fractal dimension. Fractal patterns can of course be extremely complex and involve variations in color, depth, shape and pattern. To keep the experiment to the simple focus of the fractal dimension, the surfaces produced were generated for their aesthetic neutrality (lack of association to natural or synthetic commonly recognised form). Each surface was presented in consistent simple neutral color and the base shape/function used to create the fractal pattern was consistent. The only varying element was the dimension of fractal complexity in the surface patterns (see Figure 2 for examples of some surfaces used (different levels of fractal dimension). 

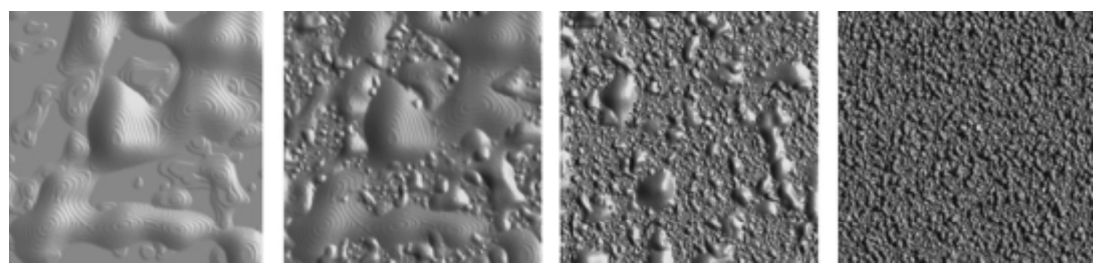

Fig. 2. Fractal surfaces used in experimental trial

The study entailed the observation and questioning of the participants regarding their interactions with these fractal surfaces. The participants were observed interacting with the visual and tactile fractal surfaces before being questioned regarding their preferences (surfaces featured 3D relief elements in physical and displacement/bump maps in visual, as result $\mathrm{D}$ values here are in the 2 to 3 range).

The participants visual preferences as recorded in the trial indicate that 18 of the 25 participants $(72 \%)$ showed a visual preference for surfaces and objects within the fractal range of $D=2.3$ to $D=2.5$. This finding matches the results from earlier studies and highlights the fact that the fractal dimension, independent of other factors such as colour, is playing a key role in the aesthetic appeal of the surface and that the simple adjustment of the fractal complexity can alter the perception of the surface significantly [5], [10], [11]. The observational findings from the study also strongly demonstrated this altered perception of the surface, caused by change in its fractal dimension. Of specific note were the correlations between response time and the observation of emotive signal, for example, the observed facial cues associated with negative responses were very strong in relation to an unseen response (virtual tactile/touch) but almost imperceptible in relation to a negative visual response. These observations also found that the unseen tactile response is usually quick, under a three second response time, especially in regard to the objects which offer a greater level of manipulation. The visual response however was usually much slower and considered. Observation of the participants showed that visual preference was given only after careful comparison and categorisation. The key difference with this study was that the fractal surfaces presented were automatically created to meet fractal dimensional values and varied only in this factor. Thus the findings indicate that the fractal dimension itself played a key role in the aesthetic appeal, or otherwise, of a surface.

\section{$4 \quad$ Applying Fractal Principles and Designs}

The contemporary built environment, in both the real world and the virtual game worlds, is dominated by the Cartesian concept of space. The world of architecture, bricks, textures, UV, tiles, panels and pavement offer a familiar rectilinear package in which to place our identities, both virtual and real. The familiarity is based upon collective experience; a mechanistic experience built brick at a time. In contrast the natural world is filled with rough edges, asymmetries, complex interactions, profound depth of scale and above all, life. Fractals and other forms of recursive patterns can 
provide rich visuals, not only in the real natural world but also in the virtual space of interactive computer games [10], [12]. In particular these mathematical patterns can often provide more natural appearing shapes and three-dimensional structural forms [5]. The use of fractal/recursive methods to automatically create complete structural game environments has been explored in several projects and this offers potential to address some issues regarding the scale of modern interactive computer games and the need for increasing numbers of new spaces and environments [3], [13].

As the results from this study indicate, people find objects and surfaces that are too simple ( $\mathrm{D}$ values $<2.25$ ) or too complex ( $\mathrm{D}$ values $>2.75$ ) to be less appealing. By quantifying this through fractal dimension/complexity, this provided a mechanism through which spaces could be designed to be more or less appealing. The second phase of this research involved not simply understanding the nature of aesthetic response to fractal dimension, but actively adjusting the fractal dimension for effect.

\subsection{Fractal Surface Designs and Complexity in Game Environments}

Applying fractal techniques to surfaces that require a more natural feel, and hence the breakup of the simple linear nature of polygonal and flat textured surfaces, into richer "self-similar" patterns has been explored in various forms. The most common examples of this include the use of fractal methods to add noise and natural disturbance to fog, dust and water patterns [4], [14]. The key focus of these uses is in natural surfaces, although the principals have potential in other areas.

The second phase of this project addressed the application of fractal patterns in surfaces, but not those used in natural features like water and fog, but instead looked at the use of fractal surfacing in the built environment. Stepping into the very heart of the rectilinear world of bricks, tiles and pavers, this research applied the concepts and knowledge gained in the earlier trials, on fractal complexity, to these built environment features. The focus of this work was on the use of surfacing techniques in the form of texture and bump maps applied using a special shading mechanism based around creating different levels of fractal complexity in the surface.

\subsection{Fractal Surface Design and Implementation}

To apply the concept of fractal complexity to the design of surfaces a tiling technique was implemented. The fractal complexity was controlled through the use of tiled textures based on a simple equilateral triangle. These tiles were different to the normal rectangular tiles used in texture maps and were instead sphinx hexiamond based tiles (consisting of six equilateral triangles arranged as in Figure 3). These tiles can be added together to make larger versions and also recursively subdivided to differing levels of complexity in a classic fractal self-similar manner.

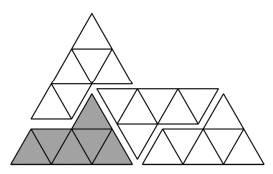

Fig. 3. Sphinx hexiamond tiles and laying patterns 
The use of alpha channel based textures, with the sphinx hexiamond based tile pattern, and a shader that transformed and re-combined them based on fractal dimension provided the ability to utilize fractal tile patterns at varying levels of fractal complexity. This level of complexity can be varied by arranging tiles in differing patterns and also by subdividing the tiles into greater levels of detail (see Figure 4 for examples).
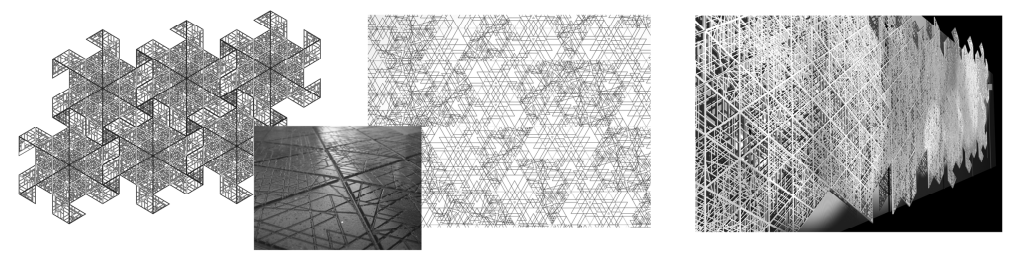

Fig. 4. Laying tiles using differing methods to achieve differing fractal complexity levels

This implementation provided a practical method, using simple tile based elements, to algorithmically create surfaces with differing levels of fractal complexity on demand. When combined with the knowledge of aesthetic preference of fractal complexity levels, this provides a means to create new surfaces of both aesthetically pleasing (D 2.3-2.5), and, perhaps for some game scenarios, aesthetically undesirable form (eg. 2.5+ for spaces to become more uncomfortable for players).

\section{$5 \quad$ Conclusions and Discussion}

Fractal patterns offer an automated mathematical mechanism to create rich patterns. These rich patterns can be applied in interactive game environments and in many cases can add a natural feel to the spaces in which they are used. The findings from this study have identified that differing levels of fractal dimension and complexity in a surface have a direct affect on the viewers comfort with that surface. Applied to games design, through the use of texture and bump maps, applied using non-standard shaders to construct the fractal complexity, this allows the designer to apply an automated mathematical approach to creating surfaces that can make the viewer more or less comfortable with the space.

\section{References}

1. Mandelbrot, B.B.: The fractal geometry of nature. Macmillan, New York (1983)

2. Mandelbrot, B.B.: Fractals: Form, change and dimension. WH Freemann and Company, San Francisco (1977)

3. Walsh, P., Prasad, G.: The use of an aesthetic measure for the evolution of fractal landscapes. In: 2011 IEEE Congress on Evolutionary Computation (CEC), pp. 1613-1619. IEEE Press, New York (2011)

4. Fan, N.: Realistic Rendering of Three-Dimensional Ocean Waves Based on Fractal. Advanced Science Letters 11(1), 469-472 (2012) 
5. Pentland, A.: Fractal-based description of natural scenes. IEEE Transactions on Pattern Analysis and Machine Intelligence 6, 661-674 (1984)

6. Bovill, C.: Fractal Geometry in Architecture and Design. Birkhäuser, Boston (1996)

7. Salingaros, N.: A scientific basis for creating architectural forms. J. Arch. Plan. Res. 15, 283-293 (1998)

8. Salingeros, N.: Connecting the Fractal City. In: Keynote Speech, 5th Biennial of Towns and Town Planners in Europe, Barcelona (2003)

9. Patterson, D.: Using Interactive 3D Game Play to Make Complex Medical Knowledge More Accessible. Procedia Computer Science 29, 354-363 (2014)

10. Spehar, B., Clifford, C., Newell, B., Taylor, R.: Universal aesthetic of fractals. Computers \& Graphics 27(5), 813-820 (2003)

11. Aks, D., Sprott, J.: Quantifying aesthetic preference for chaotic patterns. Empirical Studies of the Arts 14(1), 1-16 (1996)

12. Hendrikx, M., Meijer, S., Van Der Velden, J., Iosup, A.: Procedural content generation for games: A survey. ACM Transactions on Multimedia Computing, Communications, and Applications (TOMCCAP) 9(1) (2013)

13. Bourke, P., Shier, J.: Space Filling: A new algorithm for procedural creation of game assets. In: Proceedings of the 5th Annual International Conference on Computer Games Multimedia \& Allied Technology (2013)

14. Haase, F., Klein, M., Tarnowsky, A., Wolter, F.: Interactive fractal compositions. In: Proceedings of the 11th ACM SIGGRAPH International Conference on Virtual-Reality Continuum and its Applications in Industry, pp. 181-188. ACM Press, New York (2012) 